\title{
Development of spatial preferences for counting and picture naming
}

\author{
Birgit Knudsen • Martin H. Fischer • \\ Gisa Aschersleben
}

Received: 25 July 2014/ Accepted: 14 October 2014/Published online: 19 October 2014

(C) Springer-Verlag Berlin Heidelberg 2014

\begin{abstract}
The direction of object enumeration reflects children's enculturation but previous work on the development of such spatial preferences has been inconsistent. Therefore, we documented directional preferences in finger counting, object counting, and picture naming for children ( 4 groups from 3 to 6 years, $N=104)$ and adults $(N=56)$. We found a right-side preference for finger counting in 3to 6-year-olds and a left-side preference for counting objects and naming pictures by 6 years of age. Children were consistent in their special preferences when comparing object counting and picture naming, but not in other task pairings. Finally, spatial preferences were not related to cardinality comprehension. These results, together with other recent work, suggest a gradual development of spatial-numerical associations from early non-directional mappings into culturally constrained directional mappings.
\end{abstract}

\section{Introduction}

Numerical cognition in adults exhibits a pervasive association between numbers and space: processing small numbers (e.g., 1 or 2 ) activates left space, while processing larger numbers (e.g., 8 or 9) activates right space. This so-

B. Knudsen $(\bowtie) \cdot$ G. Aschersleben

Saarland University, Campus Gebäude A 1.3,

66123 Saarbrücken, Germany

e-mail: b.knudsen@mx.uni-saarland.de

G. Aschersleben

e-mail: aschersleben@mx.uni-saarland.de

M. H. Fischer

University of Potsdam, Karl - Liebknecht Str. 24/25,

14476 Potsdam, Germany

e-mail: martinf@uni-potsdam.de called SNARC effect (Spatial Numerical Association of Response Codes; Dehaene, Bossini, \& Giraux, 1993) is today understood to reflect a result of enculturation into a language community through reading, writing, and counting practices. Several observations support this view, whereas left-to-right reading cultures show a preference for a left-to-right mapping of numbers, right-to-left reading cultures show a preference for a right-to-left mapping of numbers (reverse-SNARC effect; Shaki, Fischer, \& Petrusic, 2009; Zebian, 2005). The directionality of the SNARC effect even varies depending on the writing direction associated with a particular number format. Among Chinese participants, Arabic numbers were mapped left-to-right, whereas Chinese number symbols were mapped bottom-to-top (Hung, Hung, Tzeng, \& Wu, 2008). In Western cultures the starting point for reading and writing a sentence, as well as for counting off object arrays, is usually on the left side, and this might impose a directional preference also on the domain of numbers, by beginning to count on the left side. It has been proposed that this culture-specific generalization eventually leads to a spatial representation of numbers on a mental number line (MNL; cf. Bachot, Gevers, Fias, \& Roeyers, 2005; Berch, Foley, Hill, \& Ryan, 1999; van Galen, \& Reitsma, 2008; White, Szúcs, \& Soltész, 2012; for a recent review see Göbel, Shaki, \& Fischer, 2011). Previous studies have provided snapshots of this hypothesized enculturation from various cultures and from different age groups who were tested on a range of tasks. The aim of the current study was to determine the age at which German preschool children first reliably count from left-to-right as well as read pictures from left-to-right, using a consistent set of spatial tasks across cohorts from within a single culture.

Spatial preferences conforming to the reading and writing direction within a given culture are already present 
in preschool age, well before being taught how to read and write. For example, Opfer, Thompson, and Furlong (2010) and Opfer and Furlong (2011) showed 4-year-old children two rows of boxes (sample and matching arrays) with seven numbered compartments each. Subsequently, a winner card was revealed from one of the compartments in the sample array and the children had to bring out the card in the same numbered compartment in the matching array. In both studies, children were faster and more accurate when the compartments were numbered left-to-right than when they were numbered right-to-left, thus revealing a preferential spatial-numerical arrangement. Moreover, the majority of children also counted objects left-to-right. More recently, de Hevia, Girelli, Addabbo, and Macchi Cassia (2014) applied the habituation paradigm to show that preverbal 7-monthold Italian infants already prefer a left-to-right increase of numerosity over a right-to-left increase. These and other recent studies raise the question about the origins of SNARC. One hypothesis is an inherited preference for relating numerosity and space. Specifically, de Hevia and Spelke (2010) hypothesize that humans may be predisposed to treat number and space as intrinsically related. In one study, preschoolers had to manually bisect lines with nonsymbolic numerical flankers (dot arrays). Results revealed that children's estimations of the lines' midpoints were systematically biased towards the larger dot arrays, evidencing an effect of perceived number onto perceived space (de Hevia \& Spelke, 2009). In a card matching game, preschoolers were further shown to match number and space (length), but not number and brightness (de Hevia, Vanderslice, \& Spelke, 2012). De Hevia and colleagues also found further evidence for an inherited preference for relating numerosity to space in infants. Eight-month-old infants perceive a correspondence between the increasing or decreasing numerosity of geometrical shapes and an increasing or decreasing sequence of spatial lengths (lines) (de Hevia \& Spelke, 2010; see also Lourenco \& Longo, 2010, for similar results). By the same age, infants show SNARC-congruent attentional shifts when presented with small and large numerosities (dots). After a small number cue, 8- to 9-month-old infants shift their attention quicker to the left than to the right and vice versa for a large number cue (Bulf, de Hevia, \& Macchi Cassia, 2014). De Hevia and colleagues concluded that the mapping between number and space is intuitive for children well before the onset of formal schooling. Although this inherited preference hypothesis provides no account for the culture-specific directionality of SNARC it is consistent with evidence for spatial-numerical mapping preferences in other species, as would be expected from an evolutionary account (cf. Adachi, 2014; Drucker \& Brannon, 2014; Gulledge, 2006; Rugani, Kelly, Szelest, Regolin, \& Vallortigara, 2010; Rugani, Vallortigara, Vallini, \& Regolin, 2011).
Alternatively, observing directional biases in adult practices such as reading, writing and counting might lead to over-generalization of this spatial concept to numbers well before learning to read in preschoolers. Consistent with this directional learning hypothesis are results of other recent studies investigating preschoolers' appreciation of number and space in a variety of tasks. In a numerosity comparison task, Patro and Haman (2012) asked 4-year-old children to indicate which of two displays, appearing on the right and on the left side of a touch screen, has more/ fewer sweets (rectangles). The children showed faster responses for fewer items on the left than on the right side and vice versa. Five-year-olds showed the same SNARClike pattern of response times in a non-symbolic magnitude comparison task in which they had to decide whether there were more or fewer cookies (brown dots) than previously presented party guests (blue dots) (Ebersbach, Luwel, \& Verschaffel, 2014). Six-year-old kindergartners' estimations on a number line (1-100) were also shown to be more accurate when the number line was oriented SNARC congruently rather than SNARC incongruently (Ebersbach, 2014). Studies investigating preschoolers' understanding of counting and counting rules in particular have found that children are significantly better at detecting actual counting errors (e.g., counts resulting in the wrong cardinal value) than pseudo-errors (Briars \& Siegler, 1984; Kamawar et al., 2010; LeFevre et al., 2006; Rodríguez, Lago, Enesco, \& Guerrero, 2013). Pseudo-errors are correct counts that violate conventional (nonessential) counting rules, such as counting items sequentially, e.g., from left-to-right (order irrelevance principle; Gelman \& Gallistel, 1978). However, whereas Briars and Siegler (1984) and LeFevre et al. (2006) found that most kindergarteners know that counting items left-to-right is unessential and, therefore, are more willing to accept reverse direction counts, Rodríguez et al. (2013) and Kamawar et al. (2010) recently found that among children's main reasons for rejecting pseudo-errors was the violation of left-to-right direction of counting, with kindergarten children favoring a left-to-right or top-tobottom direction of counting. Finally, Shaki, Fischer, and Göbel (2012) documented a preschool preference to start counting of objects according to the culture-specific reading direction, which is subsequently enhanced by the acquisition of reading habits for English and Palestinian children and reduced for Israeli children due to an emerging directional conflict between reading text right-toleft and reading numbers left-to-right.

In summary, it appears that there is a general, and possibly inherited, preference to relate magnitude concepts to space, which is subsequently shaped by culture-specific directional activities that lead to systematic counting preferences in children. These may in turn be a developmental precursor of the ubiquitous SNARC effect. The 
mechanism that mediates between these developmental stages is, however, unclear. Here, we examined a specific prediction of the directional learning hypothesis of the origin of SNARC. The observation that children commonly learn to count with their fingers suggests that finger counting habits might be a developmental precursor of SNARC. Finger counting is not only an ancient method used to contend with number and numerosities (William \& Williams, 1995), but it is also widespread across cultures (Bender \& Beller, 2012; Flegg, 1989; Göbel et al., 2011). For example, Lindemann, Alipour, and Fischer (2011) used an internet questionnaire to document a clear preference among adults from several Western countries to start counting on the left hand, consistent with the idea that their association between small numbers and left space might be a reflection of early acquired counting preferences. Unfortunately, research on directional finger counting habits in young children is rather sparse. Sato and Lalain (2008) found that the majority of 4- to 11-year-old French children started counting with their right hand and not with their left hand, a finding that conflicts with older work by Conant (1896/1960) who reported that among 206 children aged 4-8 from public schools in Worcester/Massachusetts, almost all began to count with their left hand and that this left-preference remained in an older cohort. Newman and Soylu (2014) found that $46 \%$ of American 5- to 12-yearold children started to count with their right hand. Right starters were further shown to be more accurate than leftstarters on a timed single-digit addition test, which may be due to differences in interhemispheric communication.

Previtali, Rinaldi and Girelli (2011) reported a study by Rinaldi and Girelli (2011) showing that $73 \%$ of Italian 3to 6-year-old children started to count with their right hand. Furthermore, they state that Rinaldi and Girelli (2011) did not find a relationship between directional biases of mapping numbers onto egocentric space (finger counting) and allocentric space (counting arrays of elements). However, the developmental trajectory of these measures is still unclear. Given that some studies identified finger awareness (finger gnosia) as a significant predictor of arithmetic performance in young children (Gracia-Bafalluy \& Noël, 2008; Noël, 2005; Reeve \& Humberstone, 2011) we set out to determine more precisely the age at which German preschool children first reliably consider numbers as being spatially distributed from left-to-right, how they do so with their fingers compared to other objects, and whether a leftto-right preference is continuous across age groups. We also asked whether possible trends in spatial preferences would be systematically related to number knowledge.

In the present study, 3- to 6-year-old preschool children's and adult's directional preferences were examined in three spatial tasks. In the finger counting task (FCT) participants were asked to count with their fingers; in the candle counting task (CCT) they were asked to count aloud a row of tea candles in front of them; and in the picture naming task (PNT) they were asked to name a row of pictures. FCT and CCT assessed the mapping of numbers onto egocentric and allocentric space, respectively, while PNT assessed a possible spatial bias in directional scanning induced by early reading-related experiences. Even though 3- to 6-yearold preschool children are not yet formally taught how to read or write, they rather frequently look at picture books, generally together with their parents. Thus, the PNT might re-instantiate a directional processing context that influences spatial-numerical mapping, as was previously shown in adults (e.g., Hung et al., 2008).

In addition to the spatial tasks performed by the participants, all parents of the children were asked to fill in the finger counting questionnaire first employed by Fischer (2008; see also Lindemann et al., 2011). This allowed us to examine the role of finger counting observation to directional learning. The questionnaire evaluates the explicit association between fingers and numbers and also records hand preference for everyday activities. Hand preference of children was determined with three bimanual tasks (see below).

Finally, two number tasks, the give-N task and the direction task (Sarnecka \& Carey, 2008), were administered to evaluate children's knowledge of the cardinality (give- $\mathrm{N}$ task) and the ordinality (direction task) of numbers. Two levels of such knowledge have previously been identified: Subset knowers only know the cardinal value of numbers within the subitizing range (1 through 4$)$, whereas cardinal-principle knowers know the cardinal value of all numbers within their counting range (Le Corre \& Carey, 2007; Sarnecka \& Carey, 2008; Wynn, 1990, 1992). Children who know the cardinal value of all the numbers within their counting range might be more inclined to conceive of numbers as spatially distributed as those children more likely are the more experienced counters.

\section{Method}

\section{Participants}

One hundred and four preschoolers, equally distributed across four age groups $(n=26), 182$ parents and 56 adults took part in the study (3-year-olds: 13 female, $M$ age $=40.4$ months, $\mathrm{SD}=2.74,19$ right handed, 5 ambidextrous; 4-year-olds: 10 female, $M$ age $=50.5$ months, $\mathrm{SD}=6.16,22$ right handed, 1 ambidextrous; 5 -year-olds: 14 female, $M$ age $=65.1$ months, $\mathrm{SD}=3.25,25$ right handed, 1 ambidextrous; 6-year-olds: 16 female, $M$ age $=76.7$ months, $\mathrm{SD}=4.1,24$ right handed; adults: 36 female, $M$ age $=30.6$ years, $\mathrm{SD}=9.31,51$ right handed, 1 ambidextrous; parents: 101 mothers, $M$ age $=36.6$ years, $\mathrm{SD}=5.07,97$ right handed, 81 fathers, 
$M$ age $=41.3, \mathrm{SD}=6.23,73$ right handed, 3 ambidextrous, 1 missing entry for handedness). Participants were recruited and tested in kindergartens in the Saarbrücken area of Germany and in the Developmental Psychology Unit, Saarland University, Germany. Parents and adults in the adult control group were predominantly Caucasian with an average socioeconomic background.

Four additional children were tested but their data were excluded from analyses, because the child was unwilling or too shy (one 3-year-old, two 4-year-olds) or because the testing session was not recorded (one 6-year-old). Furthermore, data from individual tasks were excluded from analyses as follows: from the give- $\mathrm{N}$ task $(n=3)$, because of experimenter error (one 3-year-old, one 4-year-old) or the child being unwilling to perform the task (one 3-yearold); from the direction task $(n=3)$, because of experimenter error (one 3-year-old) or the child being unwilling to perform the task (two 3-year-olds); and from the FCT $(n=7)$ because of parental interference (one 3-year-old), the child being unwilling or too shy (two 3-year-olds, one 4-year-old) or because the child did not know what to do (three 3-year-olds). Data from one additional 6-year-old could not be analyzed in the FCT because she counted simultaneously with both hands.

\section{Materials and procedure}

In all tasks the experimenter and the child sat opposite each other on a small door mat $(39 \times 30 \mathrm{~cm})$ on the floor at a distance of approximately one meter. Behind the experimenter a camera was placed for recording the child.

\section{Handedness}

First, handedness of each child was determined by three bimanual tasks: the child was asked, in random order, to demonstrate how to put butter on a slice of bread, how to put toothpaste on a tooth brush, and how to deal cards. The materials involved a small wooden slice of bread $(7.5 \times 5 \times 0.9 \mathrm{~cm})$ with a small wooden knife $(18 \times 1.5 \mathrm{~cm})$, a children's toothbrush with a tube of children's toothpaste and a deck of playing cards featuring different kinds of sea animals. Hand preference was assigned on the basis of the majority of hand uses for holding the knife, card or toothbrush.

\section{Spatial tasks}

Three spatial tasks were administered to assess directional preferences when counting with the fingers (Finger counting task or FCT), when counting objects (Candle counting task or CCT) and when naming an array of pictures (Picture naming task or PNT).
For the FCT a brown bear hand puppet $(24 \times 14 \mathrm{~cm})$ with fingerless paws was used. While holding up the bear the experimenter said 'Look, this is Oscar. But...you know what? Oscar can't count! Can you show Oscar how to count?'. If the child did not spontaneously use her fingers and instead started counting verbally, the experimenter stopped the child at approximately 10 and asked if the child could show Oscar with her fingers how to count so that Oscar could see how she is counting.

For the CCT an array of 10 tea candles was mounted equidistant $(2 \mathrm{~cm})$ onto white cardboard $(10 \times 56 \mathrm{~cm})$. The experimenter put the candles in front of the child and asked 'Can you count the candles for me?'.

In the PNT 5 pictures (an apple, a caterpillar, a sun, a house and a tree, $10 \times 10 \mathrm{~cm}$ each) were positioned equidistant $(3 \mathrm{~cm})$ on white cardboard $(68 \times 7 \mathrm{~cm})$. The experimenter placed all pictures simultaneously in front of the child and asked 'Can you tell me what is on the pictures?'. For all three tasks the experimenter coded whether the child started to count/name pictures from left-to-right or from right-to-left.

\section{Number tasks}

Two number tasks, the direction task and the give-N task, were administered following Sarnecka and Carey (2008) to assess children's understanding of the ordinality (direction task) and cardinality of numbers (give- $\mathrm{N}$ task).

The Direction task examined if children understand that moving forward in the numerical list represents adding items to a set, whereas moving backward represents subtracting items from a set (Sarnecka \& Carey, 2008). The task consisted of two paper plates ( $22 \mathrm{~cm}$ in diameter) and four sets of 12 items, six of one color and six of another color (green and purple pegs, black and white hair ties, red and yellow pieces of a sponge, brown and silver squared chips of card board); each set of items was only used once in four trials. The experimenter put the two plates in front of the child and said 'In this game you are not supposed to count. Instead, I will ask you a question and you have to respond right away, okay?'. Then, she put either five or six items on each plate (alternating across trials) and said 'I put five [six] pegs on this plate and five [six] pegs on this plate. Look, on both plates there are five [six] pegs, right? And now, watch what I am doing. I move one peg from this plate to this plate. And now I ask you, which plate has now four/ six [five/seven] pegs?'. If the child attempted to count the items the experimenter covered up the plates and the trial was repeated. Two trials started with five items per plate, one trial asked about four, the other trial asked about six items. The other two trials started with six items per plate, one trial asked about five, the other trial asked about seven items. Each trial was scored either correct or incorrect. 
The give- $\mathrm{N}$ task examined if children understand that numbers refer exclusively to a specific set of items (e.g., that the number ' 5 ' refers to five items). A small, stuffed toy leopard $(16 \times 4 \mathrm{~cm})$, a white, plastic bowl $(2.5 \mathrm{~cm}$ high, $12 \mathrm{~cm}$ in diameter) and a blue plastic cup $(8 \mathrm{~cm}$ high, $11 \mathrm{~cm}$ in diameter) containing ten multicolored-foam rings $(1.5 \times 0.8 \mathrm{~cm})$ were used. The experimenter lined up the leopard, his food bowl and the cup containing his treats in front of the child. Then, she told the child that the leopard is hungry and asked 'Can you give the leopard one treat?'. After the child had responded the experimenter asked the follow-up question 'Is that one?'. Next, the treats were put back into the cup and the experimenter asked the child to give three treats to the leopard, followed by 'Is that three?'. If the child responded with 'no', the request and the followup question were repeated until the child confirmed to have given the requested number of treats. Further requests depended on the child's success or failure. When the child correctly responded to a request of $N, N+1$ was requested. Conversely, when the child responded incorrectly to a request of $N, N-1$ was requested. This continued until the child had at least two successes for $N$ and two failures for $N+1$ with the maximum request being six treats. Each child's knower level was determined by the highest numeral the child responded to correctly twice. In accordance with previous research (e.g., Sarnecka \& Carey, 2008), children who succeeded up to the number word 'four' (but not at 'five') were considered subset knowers and children who succeeded at 'five' or 'six' were considered cardinal-principle knowers (CP-knowers).

Participants in the adult group also completed the FCT but only a subgroup of 26 adults completed the other spatial tasks and none performed the number tasks, due to predictable ceiling effects.

\section{Task order}

For each age group the tasks were randomized as follows: handedness was always assessed first, with the order of the handedness tasks randomized across participants. Subsequently, the spatial and number tasks were administered in counterbalanced order (except for the 26 adults who only performed the spatial tasks). The order of sub-tasks comprising the spatial tasks (finger counting, candle counting and picture naming) was randomized. Additionally, the order of pictures within the PNT was randomized across participants and the order of the two number tasks was counterbalanced. In the give-N task the side (right/left of the food bowl) of the blue plastic cup from which children picked the treats was also counterbalanced across participants. In the direction task, the direction in which the

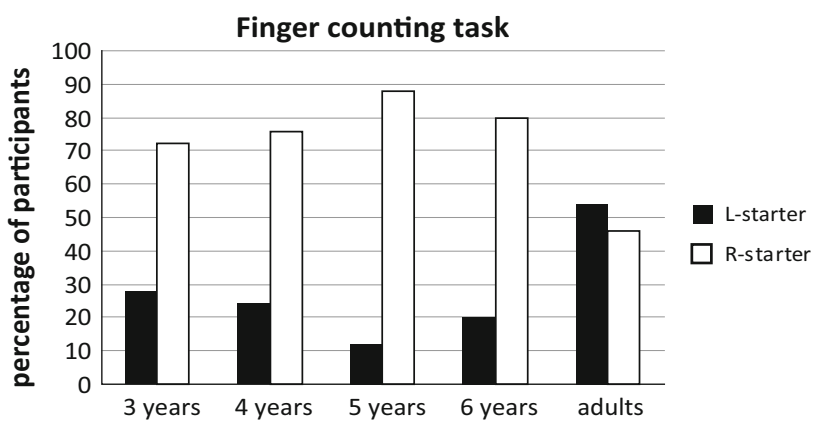

Fig. 1 Percentages of left- and right starters in the FCT in 3- to 6-year-olds and the adult subgroup

experimenter moved one item from one plate to the other was again counterbalanced such that for each participant the experimenter moved the item two times to the left and two times to the right. The starting numbers of items per plate (either five or six) as well as the following question (about the plate with fewer or more items, respectively) were counterbalanced across participants as follows: half of the participants started with five items per plate and the other half of the participants started with six items per plate. Half of the five starters (six starters) received a question about four (five) items and the other half of the five starters (six starters) received a question about six (seven) items.

\section{Parental finger counting}

Finally, we also recorded parental finger counting habits to determine whether these might influence the children's spatial preferences. Parents' $(n=182,101$ mothers, 97 right handed; 81 fathers, 73 right handed, 3 ambidextrous) directional preference when counting with the fingers was assessed using the finger counting questionnaire employed by Fischer (2008). A piece of paper showed two open hands, denoted 'left' and 'right', respectively, palms facing the participant with the thumbs pointing outward and the other fingers upward. The participant was asked to imagine to count with her fingers from 1 to 10 and to write the numbers above each corresponding finger of the left and the right hand on the paper.

\section{Results}

For all analyses, non-parametric tests were used with a significance level of $\alpha=0.05$. If not denoted otherwise, all $p$ values reported are exact and two tailed. In all comparisons of children and adults, the results of the adult subgroup that completed all three spatial tasks were compared to the results of the children's group. 
Table 1 Results of the spatial and number tasks

\begin{tabular}{|c|c|c|c|c|c|c|c|c|c|c|}
\hline & \multicolumn{3}{|c|}{ Finger counting task } & \multicolumn{3}{|c|}{ Candle counting task } & \multicolumn{2}{|c|}{ Picture naming task } & \multirow{2}{*}{$\begin{array}{l}\text { Direction } \\
\text { task } \\
\text { Mean score }\end{array}$} & \multirow{2}{*}{$\begin{array}{l}\text { Give-N } \\
\text { Mean } \\
\text { score }\end{array}$} \\
\hline & $\mathrm{L}$ starter & $\mathrm{R}$ starter & $\begin{array}{l}\text { Mean } \\
\text { correct }\end{array}$ & L starter & $\mathrm{R}$ starter & $\begin{array}{l}\text { Mean } \\
\text { correct }\end{array}$ & $\mathrm{L}$ starter & $\mathrm{R}$ starter & & \\
\hline 3 years & $28 \%(5)$ & $72 \%(13)$ & 4.11 & $42 \%(11)$ & $58 \%(15)$ & 6.46 & $62 \%(16)$ & $38 \%(10)$ & 1.91 & 2.42 \\
\hline 4 years & $24 \%(6)$ & $76 \%(19)$ & 8.67 & $62 \%(16)$ & $38 \%(10)$ & 9.84 & $50 \%(13)$ & $50 \%(13)$ & 3.04 & 5.44 \\
\hline 5 years & $12 \%(3)$ & $88 \%(23)$ & 9.96 & $58 \%(15)$ & $42 \%$ & 9.88 & $62 \%(16)$ & $38 \%(10)$ & 3.58 & 5.81 \\
\hline 6 years & $20 \%(5)$ & $80 \%(20)$ & 10 & $73 \%(19)$ & $27 \%(7)$ & 10 & $69 \%(18)$ & $31 \%(8)$ & 3.69 & 5.96 \\
\hline Adults & $54 \%$ & $46 \%$ & 10 & $100 \%(26)$ & $0 \%(0)$ & 10 & $100 \%(26)$ & $0 \%(0)$ & & \\
\hline
\end{tabular}

Entries for left-starter and right-starter columns are percentages of participants (with number of participants in brackets)

Spatial tasks

\section{Finger counting task}

As depicted in Fig. 1, in the FCT, the distribution of leftstarters and right starters among 3- to 6-year-old children did not change significantly across age, $\chi^{2}(3,94)=2.08$, $p=0.572$. However, in contrast to the adult subgroup, the majority of children in all age groups $(>72 \%)$ were right starters, $\chi^{2}(4,120)=13.23, p=0.009, \varphi=0.33$ (see Table 1). Among right starters $(n=87) 80$ participants were right handed, 4 were left-handed and 3 were ambidextrous. Among left-starters $(n=33) 25$ participants were right handed, 5 were left-handed and 3 were ambidextrous. All children started finger counting either with their right or with their left thumb.

Finger counting skills increased with age [KruskalWallis (asymptotic), $p<0.001$ ], up to the maximum count of 10: 4-year-olds counted higher than 3-year-olds (MannWhitney $U, p<0.001), 5$-year-olds counted higher than 4-year-olds (Mann-Whitney $U, p=0.003$ ), and 5- and 6-year-olds counted about equally high (see Table 1).

\section{Candle counting task}

In the CCT, all children spontaneously started counting either on the left or right side of the array. In contrast to the preschoolers, all adults counted the candles from left-toright, $\chi^{2}(4,130)=33.79, \quad p<0.001, \varphi=0.51 \quad$ (see Fig. 2). The ratio of $\mathrm{L}-\mathrm{R}$ to $\mathrm{R}-\mathrm{L}$ counting among 3- to 6 -year-olds did not change reliably across age, $\chi^{2}(3$, $104)=5.19, p=0.158$. However, 6 -year-olds counted the candles significantly more often $\mathrm{L}-\mathrm{R}$ than $\mathrm{R}-\mathrm{L}$ (Binominal, $p=0.029$ ). Consistent with FCT, the older children counted more candles correctly [Kruskal-Wallis (asymptotic), $p<0.001]$. Four-year-olds counted more candles correctly than 3-year-olds (Mann-Whitney $U, p<0.001$ ), and about as many as 5- and 6-year-olds (Mann-Whitney $U, p$ 's $>0.349$ ). For 3-year-olds, there was a significant effect of order (Mann-Whitney $U, p=0.002$ ): children

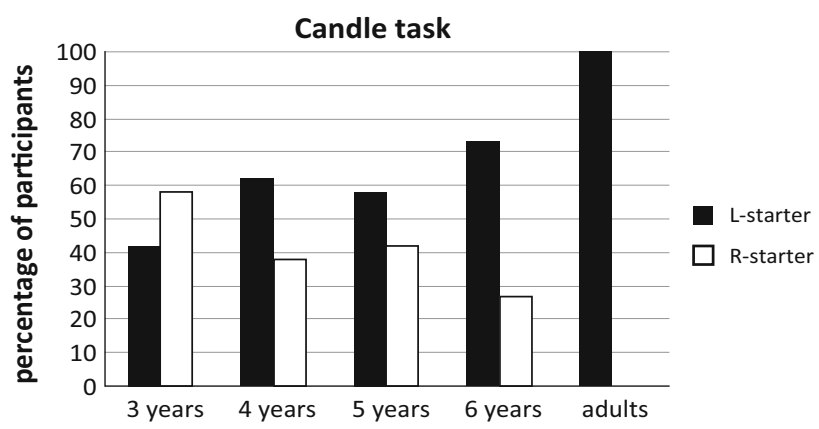

Fig. 2 Percentages of left- and right starters in the CCT in 3- to 6-year-olds and the adult subgroup

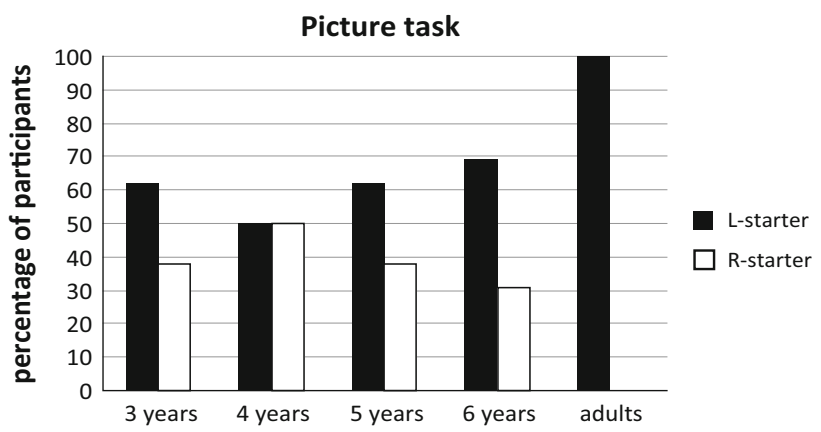

Fig. 3 Percentages of left- and right starters in the PNT in 3- to 6-year-olds and the adult subgroup

counted more candles correctly when they had done the number tasks first $(M=8.54)$ than when they had done the spatial tasks first $(M=4.38)$.

Comparing FCT and CCT, both 3- and 4-year-olds were able to count more candles than fingers (Wilcoxon, $p=0.001 ; p=0.008$, for the two ages groups, respectively).

\section{Picture naming task}

In the PNT, all but 3 children (two 4-year-olds, one 5-yearold) spontaneously began to name pictures from either the left or right side; those 3 children did too on their second 
attempt. In contrast to the 3- to 6-year-olds, all adults named the pictures $\mathrm{L}-\mathrm{R}, \chi^{2}(4,130)=32.52, p<0.001$, $\varphi=0.5$ (see Fig. 3). The ratio of $\mathrm{L}-\mathrm{R}$ to $\mathrm{R}-\mathrm{L}$ picture naming in 3 - to 6-year-olds did not change significantly across age $\chi^{2}(3,104)=2.05, p=0.616$. However, there was a trend in the group of 6-year-olds to name pictures more often from the left than from the right (Binominal, $p=0.076$ ).

\section{Consistency across spatial tasks}

Given our interest in the development of spatial preferences across tasks, per age group we tested children's consistency (starting both tasks from the left, from the right side, or inconsistent, that is, one task from the left and the other from the right side) across spatial task pairings (FCT vs. CCT, FCT vs. PNT and CCT vs. PNT) against a random distribution. Consistency measures did not differ significantly from what would be expected by chance, except for the consistency in the CCT and the PNT: whereas 3-yearolds' consistency was only marginally significant $\left(\chi^{2}\right.$, $p=0.064), 4-, 5-$, and 6-year-olds were consistent above chance $\left(\chi^{2}, p\right.$ 's $\left.<0.005\right)$.

\section{Number tasks}

\section{Direction task}

In the direction task the number of correct trials increased with age [Kruskal-Wallis (asymptotic), $p<0.001$ ], with significant increases between 3- and 4-year-olds (MannWhitney $\mathrm{U}, p<0.001$ ) and 4- and 5-year-olds (MannWhitney $U, p=0.03$ ). Interestingly, for 3-year-olds there was a trend to be correct more often when items were arranged SNARC congruently (fewer items on the left than on the right plate) compared to when they were arranged SNARC incongruently (more items on the left than on the right plate): children were correct in $75 \%$ (30/48) of SNARC-congruent trials, but only $30 \%(14 / 46)$ of SNARC-incongruent trials (Wilcoxon, $p=0.073$ ).

\section{Give-N task}

In the give- $\mathrm{N}$ task, performance improved across age (Kruskal-Wallis, $p<0.001$ ), with a significant difference between 3- and 4-year-olds (Mann-Whitney $U, p<0.001$ ). For 3- and 4-year-olds, knower level was significantly correlated with age [Kendall's tau-b (asymptotic), $p<0.001]$. In line with previous research, the majority of 3 -year-olds $(88 \%)$ were subset knowers, whereas the majority of 4-year-olds $(88 \%)$ were cardinal-principle knowers (as were $96 \%$ of the 5-year-olds, and all of the 6-year-olds). Further, among 3-and 4-year-olds, cardinal- principle knowers were correct more often in the direction task compared to subset knowers, Mann-Whitney $U$, $p<0.001$. However, knower level among the two youngest age groups was not related to spatial preferences in the spatial tasks, FCT, CCT or PNT, $\chi^{2}$, all $p$ 's $>0.571$.

Adult finger counting preferences

All mothers and fathers started finger counting with their left or their right thumb, except for one mother and two fathers, who started finger counting with their index finger. Of the 182 mothers and fathers who filled in the finger counting questionnaire there were 87 left-starters $(70 \%$ mothers) and 95 right starters (42\% mothers), with significantly more left-starters among mothers than fathers $(60$ vs. $32 \%), \chi^{2}(1,182)=14.43, p<0.001, \varphi=0.28$. In contrast, of the 56 participants in the adult control group who did the finger counting task by pantomime demonstration there were 25 (60\% female) left-starters and 31 (68\% female) right starters with no significant difference between females and males, $\chi^{2}(1,56)=369, p=0.586$. Thus, the overall proportion of left-starters across both, male and female, was 48 and $45 \%$ for questionnaire and pantomime, respectively, but the proportions differed significantly among males and females, depending on how the task was administered (questionnaire vs. demonstration).

Neither mothers' nor fathers' finger counting direction was correlated with children's finger counting preference, Phi coefficients $<0.046, p$ 's $>0.768$.

\section{Discussion}

This study investigated the prediction of the directional learning hypothesis that finger counting preferences might contribute to the development of spatial-numerical associations in preschoolers. Its further goals were (a) to describe developmental patterns in directional preferences also for counting and picture naming, (b) to relate these spatial behaviors to the preschooler's understanding of counting principles, and (c) to identify consistent trends across early development.

Aside from a general improvement in all tasks with age, the following main results were obtained. First, we found clear directional spatial-numerical associations at an early age: In both FCT and CCT all children started counting either with the left thumb/candle or with the right thumb/ candle, never at any other finger/position. This observation is in line with previous studies (Briars \& Siegler, 1984; Opfer \& Furlong, 2011; Opfer \& Thompson, 2006; Opfer et al., 2010; Sato \& Lalain, 2008; Shaki et al., 2012).

Next, while previous work had established a tendency to begin to count on the left hand in adults (cf. Fischer, 2008; 
Lindemann et al., 2011) the present data reveal that the majority of children in all age groups started counting on the right hand, which is in line with the findings of Sato and Lalain (2008) and point to a strong directional influence of reading/writing acquisition on spatial-numerical associations during childhood. This result was not due to hand orientation of the children, as might be predicted from the recent results by Riello and Rusconi (2011). These authors showed that when responding to numbers with two fingers of one hand, then the SNARC effect obtains with the left hand only in a palm-up orientation and with the right hand only in a palm-down orientation, i.e., when the thumb (conventionally representing a small number) is pointing left and is thus spatially congruent with an allocentric MNL. However, post hoc video coding revealed that our finding of right-start preferences in preschoolers went together with palm-up posture preferences during finger counting: The percentage of children counting with their palms up increased from $56 \%$ among the 3-year-olds to 96-100\% among the older children. Furthermore, finger count preferences were not related to counting skills, as indexed by knower level in the give- $\mathrm{N}$ task and also parental finger count preferences were not correlated to their children's finger count preferences.

Recent evidence suggests that finger counting preferences in adults are significantly influenced by situated factors, such as performing the task by filling in the finger counting questionnaire as the parents in this study did, or spontaneously by pantomime as the children and the adult group did (Wasner, Moeller, Fischer, \& Nuerk, 2014). Like in our sample, these authors found that female participants were particularly less likely to start with their left hand when asked to count with their fingers spontaneously than when filling in the questionnaire. Female participants, therefore, seem to be more susceptible to a situated influence of the writing direction involved in filling in the questionnaire than men. Nevertheless, even if parents' finger count preferences in this study would have been assessed through spontaneous pantomime, the correlation between children's and mothers' finger count preferences, most probably, would not have turned out to be significant either. It is likely that mothers' finger count preferences in a pantomime task would have yielded only a moderately higher right start preference, comparable to the right start preference among female participants in the adult group. The right-start preference in children, however, still was considerably higher as compared to the right start preference of the female participants in the adult group. Together, these findings thus suggest that finger counting habits may not be an influential developmental precursor of SNARC.

Next, our study revealed both spatial associations and dissociations: FCT showed an early right-start preference, which was not present in the adult group. As suggested by earlier work (see Lindemann et al., 2011), this age-related difference might reflect the gradual acquisition of culturespecific preferences through schooling. For example, while most Western children tend to draw and write with their dominant right hand, they might simultaneously start to use their free left hand for counting on their fingers. If so, this process might occur later in German compared to American children (cf. the work of Opfer and colleagues, discussed again below). In contrast, PNT and CCT both showed a gradual trend towards the adult-like left start preference. Thus, in line with the findings of Rinaldi and Girelli (2011, cited in Previtali et al., 2011), allocentric tasks (such as PNT and CCT) might develop different spatial preferences than egocentric tasks (such as FCT). On the other hand, performance on CCT exceeded that on FCT, suggesting that CCT might have been easier. The fact that most children significantly differed in their directional preferences in these two tasks might reflect the difficulty in performing the task, rather than inconsistent spatial preferences for mapping numbers onto egocentric or allocentric space, respectively. Indeed, to count with the fingers poses higher motor demands on young children as compared to pointing to external objects. Young children have a hard time extending their fingers individually, while they effortlessly point to things from about 12 months of age (Knudsen \& Liszkowski, 2012; Tomasello, Carpenter, \& Liszkowski, 2007; Woodward \& Guajardo, 2002). Therefore, the present children might have started finger counting with the more dexterous hand, which almost exclusively was the preferred right hand for all children. Additional evidence for this interpretation comes from the study by Sato and Lalain (2008), who found that finger counting correlated significantly with hand preference (but see Fischer, 2008; Lindemann et al., 2011).

Four further aspects of our results deserve discussion. First, CCT results revealed that the proportion of left-toright and right-to-left counting did not differ across age. When tested against chance individually, only 6-year-olds were found to count the candles significantly more often from the left than from the right side. Surprisingly, and despite comparable sample sizes, this is not in line with the results reported by Opfer and Thomson (2006), Opfer et al. (2010), Opfer and Furlong (2011). Next to the beneficial effect of a left-to-right ordering of numbers in their search task, they reported $73 \%$ of preschoolers as well as $98 \%$ (Opfer \& Thompson, 2006) and $73 \%$ (Opfer \& Furlong, 2011) of 4-year-old children to reliably count items from left-to-right. In contrast, only $56 \%$ of the 3- to 6-year-old preschoolers in the present study started counting the candles left-to-right, with a reliable shift towards a left-toright orientation at only 6 years of age. Since the counting tasks in the present study and in the studies by Opfer and 
colleagues were very similar (counting candles versus counting pictures or poker chips), it is unlikely that the difference in the onset of a reliable left-to-right orientation in counting can be attributed to task demands. Clearly, counting objects reliably from left-to-right seems to occur later in our sample compared to Opfer et al.'s (see also Shaki et al., 2012).

Second, based on their results, Opfer et al. (2010), Opfer and Furlong (2011) propose that a left-to-right orientation in preschoolers' conception of numbers is instantiated by early practice in counting, rather than by reading experience. The results of the present study do not support this interpretation. Whereas knower level with regard to the cardinality of number was related to performance in the other number task (DT), knower level was not related to spatial preferences in any of the spatial tasks in 3- and 4-year-old children. Furthermore, children showed a reliable shift towards a left to-right orientation only by 6 years of age. At that age, children commonly already are experienced counters and have extensive experience of looking at children's books. Indeed, children's spatial preferences in the PNT were comparable with their spatial biases in the CCT. There was no difference between the ratios of left-toright and right-to-left orientation in the CCT compared to the PNT and spatial preferences co-emerged in both tasks by 6 years of age.

Third, we found in the direction task that 3-year-olds tended to be correct more often when the items on the plates were distributed SNARC congruently. This is similar to the beneficial effect of SNARC-congruent stimuli in the task studied by Patro and Haman (2012) and Opfer et al. (2010), Opfer and Furlong (2011). But how can we reconcile the finding that 3-year-olds tended to benefit from a SNARC-congruent distribution of items in the direction task with the absence of a SNARC-congruent counting behavior in the CCT? One interesting possibility is that the direction task and the CCT tap into different processes: in the direction task children's responses were based on a visual presentation of either a SNARC-congruent or SNARC-incongruent distribution of items. In contrast, in the CCT, the items were equally distributed on a horizontal plane. The presentation of a SNARC-incongruent distribution of items in the direction task might have violated children's implicit expectation of items being distributed with fewer items on the left than on the right, whereas children in the CCT had to produce a spatial response themselves. That is, the direction task might tap into early perceptual processes, whereas the CCT might tap into production processes. One possibility that follows from this observation is that SNARC-congruent expectations with regard to number might develop first and translate into action later in development. This interpretation is supported by the findings of Opfer and Furlong (2011), who examined a similar distinction. They found that children's difficulties in their search task arose as a result of spatial biases in the encoding process (expecting the sample boxes to be numbered SNARC congruently), rather than as the result in spatial biases in performance (children automatically count the matching boxes left-to-right).

Finally, 3-year-olds counted twice as many candles correctly when they had done the number tasks (direction task, give-N task) first than when they had not. This substantial enhancement of performance in CCT when assessed later during the experimental protocol could, of course, reflect a mere familiarization with the experimental procedures of the study and a resulting performance increase. However, this explanation cannot account for the selective improvement only in CCT and not in the other tasks. We, therefore, suggest that CCT improvement following the number tasks might instead reflect a spatial cueing benefit from performing in two other allocentrically coded task. Specifically, both the direction task and the give- $\mathrm{N}$ task require explicit magnitude manipulations in allocentric coordinates, which may have assisted the 3-year-olds with their subsequent performance in the equally allocentric CCT by inducing a spatial task set.

How do these results inform the ongoing debate about the origin of SNARC? The absence of a correlation between directional preferences of parents and their children speaks against an origin of SNARC through finger counting practise as predicted by the directional learning hypothesis. However, the results of the CCT and the PNT do support the directional learning hypothesis in that they show that a left bias in object counting and picture naming in the present sample of German preschoolers co-occurs only by 6 years of age.

Even though the current study does not inform about the inherited preference hypothesis, we think that the development of spatial-numerical associations might best be characterized as a multi-stage learning process that builds on a genetic predisposition to associate space and numbers, as documented by the work of de Hevia and colleagues (de Hevia \& Spelke, 2010; de Hevia et al., 2012). While this predisposition is consistent with neuroscientific theorizing such as $A$ Theory Of Magnitude (ATOM: Bueti \& Walsh, 2009; Dehaene \& Brannon, 2011; Walsh, 2003), according to which common cortical substrate is allocated to the representation of magnitudes across domains, it merely supports non-directional associations between space and number (but see de Hevia et al., 2014). A second, culturespecific process builds on this predisposition of children to establish directional habits. Specifically, preschool children live in environments with consistent directional preferences during sequential activities such as reading, writing and counting. Through observation and imitation the children learn to incorporate these directional actions into their 
own behavioral repertoire, although parental finger counting habits seem to play only a minor role in this shaping process. Instead, cultural practices are relevant, and the time course of enculturation may well differ across cultures, with German children apparently lagging behind American children with respect to the acquisition of spatial preferences in counting.

Acknowledgments We would like to thank the parents and children and the students who participated in this study. We also would like to thank Ingo Besserdich for his assistance in collecting and coding the data. We would further like to thank two anonymous reviewers for their constructive and helpful comments on an earlier version of the article. Martin H. Fischer was supported through DFG Grant FI 1915/2-1 on "Manumerical cognition".

\section{References}

Adachi, I. (2014). Spontaneous spatial mapping of learned sequence in chimpanzees: evidence for a SNARC-like effect. PLoS ONE, 9, e90373.

Bachot, J., Gevers, W., Fias, W., \& Roeyers, H. (2005). Number sense in children with visuospatial disabilities: orientation of the mental number line. Psychology Science, 47, 172-183.

Bender, A., \& Beller, S. (2012). Nature and culture of finger counting: diversity and representational effects of an embodied cognitive tool. Cognition, 124, 156-182.

Berch, D. B., Foley, E. J., Hill, R. J., \& Ryan, P. M. (1999). Extracting parity and magnitude from Arabic numerals: developmental changes in number processing and mental representation. Journal of Experimental Child Psychology, 74, 286-308.

Briars, D. J., \& Siegler, R. S. (1984). A featural analysis of preschoolers' counting knowledge. Developmental Psychology, 20, 607-618

Bueti, D., \& Walsh, V. (2009). The parietal cortex and the representation of time, space, number and other magnitudes. Philosophical Transaction of the Royal Society-Biological Section, 364, 1831-1840.

Bulf, H., de Hevia, M. D., \& Macchi Cassia, V. (2014). Do numbers orient visual attention in infants? Evidence from an eye-tracking study. Berlin: Poster presented at the International Conference on Infant Studies.

Conant, L. L. (1896/1960). Counting. In J. R. Newman, (Ed). The world of mathematics, vol. I (pp. 432-441). London: George Allen and Unwin Ltd.

Dehaene, S., Bossini, S., \& Giraux, P. (1993). The mental representation of parity and numerical magnitude. Journal of Experimental Psychology: General, 122, 371-396.

Dehaene, S., \& Brannon, E. (2011). Space, time, and numbers. Oxford: University Press.

de Hevia, M. D., Girelli, L., Addabbo, M., \& Macchi Cassia, V. (2014). Human infants' preference for left-to-right oriented increasing numerical sequences. PLOS ONE, 9, e96412.

de Hevia, M. D., \& Spelke, E. S. (2010). Number-space mapping in human infants. Psychological Science, 21, 653-660.

de Hevia, M. D., \& Spelke, E. S. (2009). Spontaneous mapping of number and space in adults and young children. Cognition, 110, 198-207.

de Hevia, M. D., Vanderslice, M., \& Spelke, E. S. (2012). Crossdimensional mapping of number, length and brightness by preschool children. PLOS ONE, 7, e35530.

Drucker, C. B., \& Brannon, E. M. (2014). Rhesus monkeys (Macaca mulatta) map number onto space. Cognition, 132, 57-67.
Ebersbach, M. (2013). Evidence for a spatial-numerical association in kindergartners using a number line task. Journal of Cognition and Development. doi:10.1080/15248372.2013.805134

Ebersbach, M., Luwel, K., \& Verschaffel, L. (2014). Further evidence for a spatial-numerical association in children before formal schooling. Experimental Psychology, 61, 323-329.

Fischer, M. H. (2008). Finger counting habits modulate spatialnumerical associations. Cortex, 44, 386-392.

Flegg, G. (1989). Numbers through the ages. London: Macmillan, Open University.

Gelman, R., \& Gallistel, C. R. (1978). The child's understanding of number. Cambridge: Harvard University Press.

Göbel, S. M., Shaki, S., \& Fischer, M. H. (2011). The cultural number line: a review of cultural and linguistic influences on the development of number processing. Journal of Cross-Cultural Psychology, 42, 543-565.

Gulledge, J. P. (2006). Hemispheric differences in numerical cognition: a comparative investigation of how primates process numerosity. Psychology Dissertations. Paper 12. http://digitalarc hive.gsu.edu/psych_diss/12.

Gracia-Bafalluy, M., \& Noël, M. P. (2008). Does finger training increase young children's numerical performance? Cortex, 44, 368-375.

Hung, Y.-H., Hung, D. L., Tzeng, O. J.-L., \& Wu, D. H. (2008). Flexible spatial mapping of different notations of numbers in Chinese readers. Cognition, 106, 1441-1450.

Kamawar, D., LeFevre, J., Bisanz, J., Fast, L., Skwarchuk, S. L., Smith-Chant, B. L., et al. (2010). Knowledge of counting principles: how relevant is order irrelevance? Journal of Experimental Child Psychology, 105, 138-145.

Knudsen, B., \& Liszkowski, U. (2012). One-year-olds warn others about negative action outcomes. Journal of Cognition and Development, . doi:10.1080/15248372.2012.689387.

Le Corre, M., \& Carey, S. (2007). One, two, three, four, nothing more: an investigation of the conceptual sources of the verbal counting principles. Cognition, 105, 395-438.

LeFevre, J., Smith-Chant, B. L., Fast, L., Skwarchuk, S., Sargla, E., Arnup, J. S., et al. (2006). What counts as knowing? The development of conceptual and procedural knowledge of counting from Kindergarten to Grade 2. Journal of Experimental Child Psychology, 93, 285-303.

Lindemann, O., Alipour, A., \& Fischer, M. H. (2011). Finger counting habits in Middle-Eastern and Western individuals: an online survey. Journal of Cross-Cultural Psychology, 42, 566-578.

Lourenco, S. F., \& Longo, M. R. (2010). General magnitude representation in human infants. Psychological Science, 21, 873-881.

Newman, S. D., \& Soylu, F. (2014). The impact of finger counting habits on arithmetic in adults and children. Psychological Research, 78, 549-556.

Noël, M. E. (2005). Finger gnosia: a predictor of numerical abilities in children? Child Neuropsychology, 11, 413-430.

Opfer, J. E., \& Furlong, E. E. (2011). How numbers bias preschoolers' spatial search. Journal of Cross-Cultural Psychology, 42, 682-695.

Opfer, J. E., Thompson, C. A., \& Furlong, E. E. (2010). Early development of spatial-numeric associations: evidence from spatial and quantitative performance of preschoolers. Developmental Science, 13, 761-771.

Opfer, J. E., \& Thompson, C. A. (2006). Even early representations of numerical magnitude are spatially organized: Evidence from a directional magnitude bias in pre-reading preschoolers. In R. Sun $\&$ N. Miyake (Eds.), Proceedings of the 28th annual cognitive science society (pp. 639-644). Mahwah: Erlbaum.

Patro, K., \& Haman, M. (2012). The spatial-numerical congruity effect in preschoolers. Journal of Experimental Child Psychology, 111, 534-542. 
Previtali, P., Rinaldi, L., \& Girelli, L. (2011). Nature of nurture in finger counting: a review on the determinants of the direction of number-finger mapping. Frontiers in Psychology, 2, 363.

Reeve, R. A., \& Humberstone, J. (2011). Five- to 7-year-olds' finger gnosia and calculation abilities. Frontiers in Psychology, 2, 359.

Riello, M., \& Rusconi, E. (2011). Unimanual SNARC effect: hand matters. Frontiers in Psychology, 2, 372.

Rinaldi, L., \& Girelli, L. (2011). The role of number-space associations in preschoolers' numerical competence. Beer Sheva: Poster Presented at the Workshop in Typical and Atypical Neurocognitive Aspects of Numerical Processing.

Rodríguez, P., Lago, M. O., Enesco, I., \& Guerrero, S. (2013). Children's understandings of counting: detection of errors and pseudoerrors by kindergarten and primary school children. Journal of Experimental Child Psychology, 114, 35-46.

Rugani, R., Kelly, D. M., Szelest, I., Regolin, L., \& Vallortigara, G. (2010). Is it only humans that count from left to right? Biology Letters, 6, 290-292.

Rugani, R., Vallortigara, G., Vallini, B., \& Regolin, L. (2011). Asymmetrical number-space mapping in the avian brain. Neurobiology of Learning and Memory, 95, 231-238.

Sarnecka, B. W., \& Carey, S. (2008). How counting represents number: what children must learn and when they learn it. Cognition, 108, 662-674.

Sato, M., \& Lalain, M. (2008). On the relationship between handedness and hand-digit mapping in finger counting. Cortex, 44, 393-399.

Shaki, S., Fischer, M. H., \& Göbel, S. M. (2012). The origin of number-space associations: a comparative study of spatially directional counting biases in cultures with different reading directions. Journal of Experimental Child Psychology, 112, 275-281.
Shaki, S., Fischer, M. H., \& Petrusic, W. M. (2009). Reading habits for both words and numbers contribute to the SNARC effect. Psychonomic Bulletin \& Review, 16, 328-331.

Tomasello, M., Carpenter, M., \& Liszkowski, U. (2007). A new look at infant pointing. Child Development, 78, 705-722.

Van Galen, M. S., \& Reitsma, P. (2008). Developing access to number magnitude: a study of the SNARC effect in 7- to 9-yearolds. Journal of Experimental Child Psychology, 101, 99-113.

Walsh, V. (2003). A theory of magnitude: common cortical metrics of time, space and quantity. Trends in Cognitive Sciences, 7, 483-488.

Wasner, M., Moeller, K., Fischer, M. H., \& Nuerk, H.-C. (2014). Aspects of situated cognition in embodied numerosity: the case of finger counting. Cognitive Processing,. doi:10.1007/s10339014-0599-z.

White, S., Szücs, D., \& Soltesz, F. (2012). The integration of magnitude and spatial representations in children aged 6 to 8 years. Frontiers in Psychology, 2, 392.

William, B. P., \& William, R. S. (1995). Finger numbers in the grecoroman world and the early middle ages. Isis, 86, 587-608.

Woodward, A. L., \& Guajardo, J. J. (2002). Infants' understanding of the point gesture as an object-directed action. Cognitive Development, 17, 1061-1084.

Wynn, K. (1990). Children's understanding of counting. Cognition, 36, 155-193.

Wynn, K. (1992). Children's acquisition of the number words and the counting system. Cognitive Psychology, 24, 220-251.

Zebian, S. (2005). Linkages between number concepts, spatial thinking, and directionality of writing: The SNARC effect and the reverse SNARC effect in English and Arabic monoliterates, biliterates, and illiterate Arabic speakers. Journal of Cognition and Culture, 5, 165-190. 